\title{
PUFAs, A DIETARY SUPPLEMENT AND SEIZURE THRESHOLD
}

Investigators at the University of Toronto, Canada studied the effects of chronic dietary supplementation with omega-3 polyunsaturated fatty acids (PUFAs) derived from fish oil on seizure thresholds in the amygdala as well as on blood and brain PUFA levels in 60-day-old rats. The acute effects of omega-3 PUFAs-eicosapentaenoic acid (EPA) and docosahexaenoic acid (DHA) were also tested in laboratory rats with experimental pentylenetetrazol seizures. Fish oil supplementation significantly increased amygdaloid afterdischarge thresholds after 3, 5, and 7 months treatment. Fish oil supplementation also increased serum EPA and DHA concentrations. DHA significantly increased the latency to seizure onset in the pentylenetetrazol seizure model, whereas EPA had no significant effect. (Taha AY, Trepanier M-O, Ciobanu FA, et al. A minimum of 3 months of dietary fish oil supplementation is required to raise amygdaloid afterdischarge seizure thresholds in rats - implications for treating complex partial seizures. Epilepsy Behav 2013 Apr;27(1):49-58). (Response: Dr Ameer Y Taha at National Institute on Aging, NIH, Bethesda, MD 20892. E-mail: a.taha@utoronto.ca).

COMMENT. Omega-3 PUFAs raise the seizure threshold in animals, but several months dietary supplementation may be required to demonstrate an effect in humans.

\section{METABOLIC DISORDERS}

\section{MEDNIK SYNDROME: A DEFECT OF COPPER METABOLISM}

Investigators at Bambino Gesu Children's Hospital, Rome, and other centers in Italy, Canada and France report an 8-year-old female Sephardic-Jewish patient with MEDNIK syndrome associated with a new AP1S1 homozygous mutation. She showed severe perturbations of copper metabolism with hypocupremia, hypoceruloplasminemia and liver copper accumulation with intrahepatic cholestasis. Treatment with zinc acetate resulted in improved clinical symptoms and liver copper metabolism. Reevaluation of 5 original French-Canadian patients with MEDNIK syndrome and AP1S1 mutations confirmed copper metabolism perturbation and hepatopathy in all patients. In the pathogenesis of MEDNIK syndrome, AP1S1 regulates intracellular copper machinery mediated by copper-pump proteins. This multisystem treatable disease combines clinical and biochemical signs of both Menkes and Wilson's diseases. (Martinelli D, Travaglini L, Drouin CA, et al. MEDNIK syndrome: a novel defect of copper metabolism treatable by zinc acetate therapy. Brain 2013 Mar;136(Pt 3):872-81). (Resp.: Dr Carlo DionisiVici, Bambino Gesu Children's Hospital, Rome, Italy. E: carlo.dionisivici@opbg.net).

COMMENT. MEDNIK syndrome is an acronym for mental retardation, deafness, neuropathy, ichthyosis, and keratodermia, and is caused by AP1S1 gene mutations. This rare autosomal recessive neurocutaneous syndrome, first reported in French-Canadian families (Montpetit A, et al. PLoS Genet 2008 Dec;4(12):e1000296), is now known to be caused by a perturbation of copper metabolism, and is treatable with zinc acetate. 